\title{
AN UAV SCHEDULING AND PLANNING METHOD FOR POST-DISASTER SURVEY
}

\author{
G.Q. Li ${ }^{\text {a }}{ }^{*}$, X.G. Zhou ${ }^{\mathrm{a}}$, J. Yin $^{\mathrm{a}}$, Q.Y. Xiao ${ }^{\mathrm{a}}$ \\ ${ }^{a}$ School of Geosciences and Info-Physics, Central South University, Changsha 410083,China - \\ Ligq168@163.com, zhouxiaog@gmail.com,934097163@qq.com, xiaoqy168@163.com
}

Technical Commission II

KEY WORDS: UAV, Disaster survey, Tracking, Scheduling

\begin{abstract}
:
Annually, the extreme climate and special geological environments lead to frequent natural disasters, e.g., earthquakes, floods, etc. The disasters often bring serious casualties and enormous economic losses. Post-disaster surveying is very important for disaster relief and assessment. As the Unmanned Aerial Vehicle (UAV) remote sensing with the advantage of high efficiency, high precision, high flexibility, and low cost, it is widely used in emergency surveying in recent years. As the UAVs used in emergency surveying cannot stop and wait for the happening of the disaster, when the disaster happens the UAVs usually are working at everywhere. In order to improve the emergency surveying efficiency, it is needed to track the UAVs and assign the emergency surveying task for each selected UAV. Therefore, a UAV tracking and scheduling method for post-disaster survey is presented in this paper. In this method, Global Positioning System (GPS), and GSM network are used to track the UAVs; an emergency tracking UAV information database is built in advance by registration, the database at least includes the following information, e.g., the ID of the UAVs, the communication number of the UAVs; when catastrophe happens, the real time location of all UAVs in the database will be gotten using emergency tracking method at first, then the traffic cost time for all UAVs to the disaster region will be calculated based on the UAVs' the real time location and the road network using the nearest services analysis algorithm; the disaster region is subdivided to several emergency surveying regions based on DEM, area, and the population distribution map; the emergency surveying regions are assigned to the appropriated UAV according to shortest cost time rule. The UAVs tracking and scheduling prototype is implemented using SQLServer2008, ArcEnginge 10.1 SDK, Visual Studio 2010 C\#, Android, SMS Modem, and Google Maps API.
\end{abstract}

\section{INTRODUCTION}

Annually, the extreme climate and special geological environments lead to frequent natural disasters, including earthquakes, floods, etc., and often result in serious casualties and enormous economic losses. Disaster monitoring and relief are considered as the most important tasks. And, the postdisaster survey and assessment is a significant technical work to reduce losses from natural disasters. Hence, some special information technology, including remote sensing and special analyst are often also employed for disaster reduction. Compared with conventional airborne or satellite remote sensing technology for disaster assessment, the Unmanned Aerial Vehicle (UAV) remote sensing means can improve the assessment work with advantages of its high precision, high flexibility, low cost, high efficiency and multi-angle imaging. Since 1990, UAV remote sensing technology has gradually taken active effect in data obtaining, geological disaster monitoring, rescuing and the loss assessment. Also, several previous studies have investigated methods of many scholars. O'Rourke put forward the vehicle routing problem (VRP) model (O’Rourke, 2001); Secrest proposed multiple traveling salesman problem (MTSP) model (Secrest, 2001); Alighanbari (Alighanbari, 2004) presented mixed integer linear programming (MILP) model; Ye proposed multi-UAV task allocation formal model (Ye, 2005); Li established a single task dynamic programming model based on event-driven principle(Li, 2008); Zhang constructed the mathematical model of task assignment problem by considering the decision variables and constraint s(Zhang, 2009), etc. Recently, some hybrid intelligence algorithms are involved in UAV scheduling. Su used division of labour mechanism of ant colony algorithm to solve CMTAP, and, designed the structure solution strategy based on competency assessment, state transition rules and the task cost according to the characteristics of collaborative task allocation (Su, 2008). Tao adopted contract net method and devised a new scheduling approach of global optimal (Tao, 2006, 2007). Yu studied a dynamic resource scheduling algorithm based on genetic algorithm (Yu, 2004). Stutzle studied the multiple UAVs scheduling algorithm based on particle swarm optimization (PSO) (Stutzle, 1997), etc.

An effective approach of UAVs tracking, scheduling and mission planning is studied in this paper. This paper is organized with 5 sections. Section 1 is introduction. We then discuss UAV tracking and scheduling post-disaster survey in Section 2. The planning method for post-disaster survey is discussed in section 3. The implementation and experimental software are presented in Section 4. Section 5 provides a summary and concludes the discussion.

\section{UAV TRACKING AND SCHEDULING}

\subsection{UAV Tracking}

Around the disaster area, based on network analysis technique and UAVs actual positions, some available nearest UAVs can be scheduled and added on to routes in progress. The UAV fleets are often dispatched outdoor, in fact. So, the knowledge of their locations and efficiency is greatly strategic, and the UAVs dispatch centres for disasters relief must continue monitoring the UAVs fleets, and obtain the position of them. Yet, how to obtain the actual location and real-time status of the UAVs is a common problem. After the UAV fleets are 
dispatched to other place, the UAVs fleet manager should continue to upload their positions and UAVs' status to the centres. With latest technology, in a smart phone, to efficiently collect consecutive locations of UAVs fleets, the centres can easily track the UAVs fleet in real-time. Employing Global Positioning System (GPS), and using GSM network, the UAVs tracking system can provide a constant approach to monitor the UAVs fleets. The tracking flow diagram is shown in figure 1 .

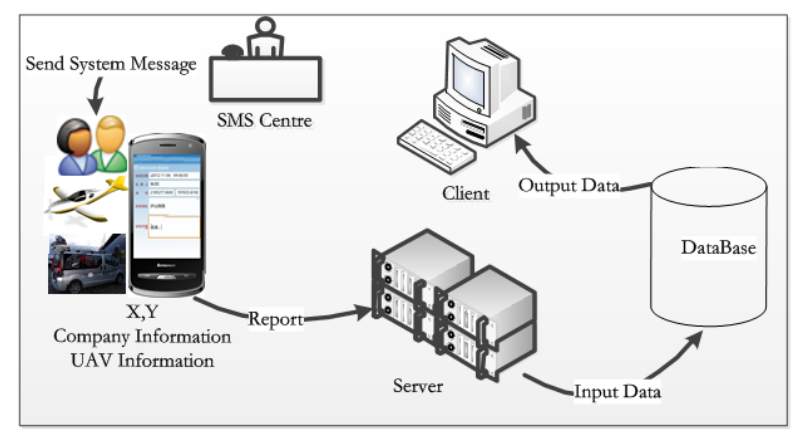

Figure 1: UAV tracking flow

To obtain the UAVs information, the steps to track the UAV are structured as follows: (1) the UAVs dispatch centre collect contacts about departments having UAVs, and store their contacts into the database; (2) the UAVs manager download and install the UAV tracking and reporting APP on their smart phones; (3) the centre administrator lists the contacts and send the requesting SMS to the UAV fleet managers, when a disaster occur; (4) after receiving the SMS, the managers open the APP and report the UAVs real-time position and status via GSM network to the centre, if they can participate the rescue execution; (5) the server receive the data from the UAV fleet and store the data into database. All these data will be used during the UAV scheduling.

\subsection{UAV Scheduling}

UAV Scheduling is to determine a few suitable UAVs to the disaster area for collecting image, with time and equipment constraints. Thus, to delineate the disaster areas and to identify the UAV gathering site are import. In order to outline the disaster area, a buffer analysis method is employed in this paper, which outlines the disaster area as a circle. Its centre is the disaster centre, and its radius is sphere of influence. Also, some crossover points, the intersection of road and the disaster affected circle, are calculated via the spatial analysis method, and are selected as the UAVs gathering sites. The figure 2 shows an instance of the gathering sites.

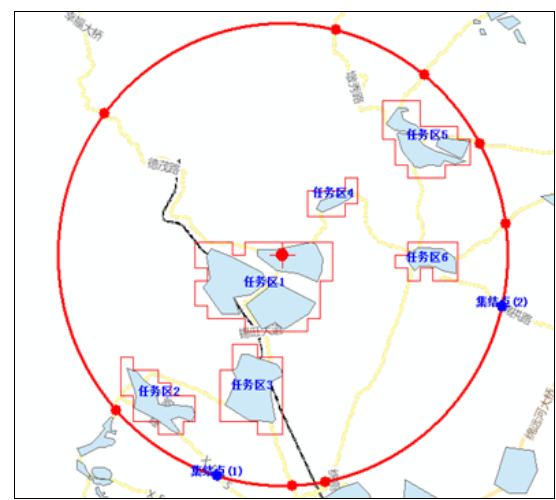

Figure 2 the UAV gathering sites
Because the huge rescue operation is urgent, the UAV arrival time (AT) from their current site to the gathering site is an important factor in UAV scheduling. In this paper, the Dijkstra algorithm is used to analysis the shortest path from the UAV site to the gathering site. According to the shortest path and the permissible speed value, the AT of UAV is calculated immediately. Furthermore, an ordered AT list of UAVs is generated rapidly. According to the list, some UAVs are chosen with their AT can meet the time constraint for the rescue.

\section{UAV TASK PLANNING}

Generally, the geological disaster affected area is very widespread, and many UAVs should been scheduled to rapidly acquits the data about the destruction. Also, in a disaster area, only a few target regions need to be monitored, such as largescale reservoirs, residential area, and import road, etc. Hence, it is vital to find a fastest solution, which can minimize the completion time, to allocate the multi-task to multi-UAV, and it should satisfy timing constraints. However, the task assignment decision is large, and growing at a non-polynomial rate, so that it is almost impossible to find the globally-optional solution. Thus, this paper investigates an approximate method that yields a fast estimate of the finishing times for the UAV to make the problem computationally tractable.

In this method, a few key regions must be outlined as tasks of UAVs firstly. Then, a suitable UAV task planning approach should be used to assign them to every UAV, and their total finishing time (TFT) should satisfy a certain time constraint. Hence, to calculate the TFT, the UAV task planning can be divided into two steps: (1) to outline these key tasks and (2) to assign them to the UAVs. In the first step, a hierarchical quadtree clustering method is investigated to identify these key tasks. In the second step, the arrival time (AT) from the UAV current site to its target task region and working hours $(\mathrm{WH})$ must be evaluated. In another word, there are a huge amount of planning schemes if there exist a lot of tasks and many available UAVs to be scheduled. The TFT of each solution is various. Among them, the selected scheme must be optimal and its TFT must satisfy a certain time constraint. Even though, it is not guaranteed that the solution is globally-optimum to the problem. In order to estimate each scheme's TFT, the $A T$ and WH must be calculated dynamically.

(1) To calculate $A T$

$A T$ is transporting hours from the rally site to its target task or from a target task to another. Perhaps, after a disaster, the roads in a certain scope are damaged. AT differs considerably from normal arrival time (NT) with unbroken road. In this paper, an influence coefficient $\Omega$ ( $\Omega: 1$ or less) is given to evaluate the AT. If $N T$ can be calculated by network analysis method, the AT can be written as:

$$
A T=N T / \Omega
$$

(2) To calculate $W H$

WH refers to the time cost, which is spent by UAV in the performing a task. It can be calculated by the UAV's capability $(U C)$, which equals the captured area per unit of time. Then, the $W H$ is shown as:

$W H=A / U C$ 
The $A$ is the task area, which can be computed easily. The $U C$ can be estimated by its maximum captured range and its maximum working speed. The maximum captured range is the scope of inspection that the camera can acquire.

Based on the $A T$ and $W H$, a UAV mission planning model is proposed to generate an optimized solution with a rescue time constraint. If $u$ denotes the number of the scheduled UAVs and $r$ denotes the number of task regions, there are 3 scenarios: (1) scenario I when $u>r$, (2) scenario II when $u=r$, and (3) scenario III when $u<r$. In order to estimate the TFT easily, we suppose that all scheduled UAVs have already arrived there rally site. For the first two of these scenarios, the UAV with greater UC will be assigned to a bigger task. The total hours may be equivalent to sum of every $W H$ with ignoring their $A T$. For the scenario III, the TFT is computed hierarchically. At the beginning, the rule to assign task region is same to the scenario I and II, and the UAV with greater $U C$ should be assigned to a bigger task. If $A T_{i}^{0}$ denotes the $A T$ of UAV $i$ to its first task region, and $W H_{i}^{0}$ denotes the $W H$ of UAV $i$ to finish its first task, then $T F T_{i}^{0}$ can be expressed as:

$$
\operatorname{TFT}_{i}^{0}=A T_{i}^{0}+W H_{i}^{0}
$$

In this phase, if the UAV $j$ is first one to complete its task, denoted as task $p$, a new task $q$ nearest to the task $p$ will be assigned to UAV $j$ after $T T H_{j}^{0}$, and $T F T j^{1}$ will be estimated by $A T_{j}^{1}$ plus $W H_{j}^{1}$. Meanwhile, the others are capturing. Next, another fastest UAV will complete its task among all working $\mathrm{UAV}$, and a new task will be allocated to it. Correspondingly, this execution will be repeated in every round until all the tasks will been allocated and completed. The following instance (figure 3) can illuminate the process described above.

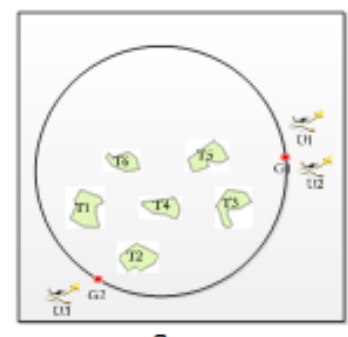

a.



C



b

d

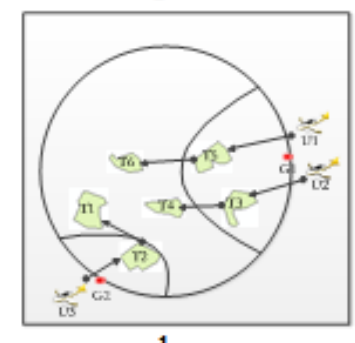

Figure 3 UAV Planning

Assume that the UAVs set $\boldsymbol{U}=\left\{U_{1}, U_{2}, U_{3}\right\}$, the rally points set $\boldsymbol{G}=\left\{G_{1}, G_{2}\right\}$, and the task set $\boldsymbol{T}=\left\{T_{1}, T_{2}, T_{3}, T_{4}, T_{5}, T_{6}\right\} . U_{1}$ and $U_{2}$ park in the rally point $G_{1}, U_{3}$ park in $G_{2}$ (figure 3a). In the first round, every $A T$ of each UAV from its rally site to the each task will be calculated via network analysis method. And every $W H$ of each UAV is estimated, too. Hence, $U_{1}$ has a matrix $\boldsymbol{M}_{1}{ }^{0}\left(\mathrm{TFT}_{1,1}{ }^{0}, \mathrm{TFT}_{1,2}{ }^{0}, \mathrm{TFT}_{1,3}{ }^{0}, \mathrm{TFT}_{1,4}{ }^{0}, \mathrm{TFT}_{1,5}{ }^{0}, \mathrm{TFT}_{1,6}{ }^{0}\right)$ in the first round. The minimum $\operatorname{TFT}_{1,5}{ }^{0}$ will be picked from $\boldsymbol{M}_{\mathbf{1}}{ }^{0}$, and the $T_{5}$ is assigned to $U_{1}$ (shown in figure 4c). Similarly, the $T_{3}$ is assigned to $U_{2}$, and $T_{2}$ is assigned to $U_{3}$. In this execution, another matrix $\boldsymbol{W}^{0}$ is generated and formed as $\left(\min \left(\boldsymbol{M}_{1}^{0}\right), \min \left(\boldsymbol{M}_{2}{ }^{0}\right), \min \left(\boldsymbol{M}_{3}{ }^{0}\right), \min \left(\boldsymbol{M}_{4}^{0}\right), \min \left(\boldsymbol{M}_{5}{ }^{0}\right), \min \left(\boldsymbol{M}_{6}{ }^{0}\right)\right)$.

Supposing that $\min \left(\boldsymbol{M}_{5}^{0}\right)$ equals $\min \left(\boldsymbol{W}^{0}\right)$, the $U_{1}$ will finish its task after $\min \left(\boldsymbol{M}_{5}{ }^{0}\right)$, and the $T_{6}$ nearest to $T_{5}$ will be allocated to $U_{1}$. So, $\boldsymbol{W}^{\mathbf{1}}$ is generated and formed as $\left(\min \left(\boldsymbol{M}_{1}{ }^{0}\right), \min \left(\boldsymbol{M}_{2}{ }^{0}\right), \min \left(\boldsymbol{M}_{3}{ }^{0}\right), \min \left(\boldsymbol{M}_{4}{ }^{0}\right), \quad \operatorname{TFT}_{1,6}{ }^{0}, \min \left(\boldsymbol{M}_{6}{ }^{0}\right)\right.$. Correspondingly, in the $\boldsymbol{W}^{\mathbf{1}}$, supposing $\min \left(\boldsymbol{M}_{2}{ }^{0}\right)$ equals $\min \left(\boldsymbol{W}^{1}\right)$, the $T_{4}$ nearest to $T_{3}$ will be assigned to $U_{2}$. At last, the $T_{4}$ is allocated to $U_{2}$ (figure $4 \mathrm{~d}$ ). So far, all tasks will be allocated and the planning scheme will be determined.

\section{EXPERIMENTAL SOFTWARE}

The previous section outlined a method of rapidly estimating completion times for individual UAV for the possible task. This section presents software achieve of the UAV scheduling and planning software. The figure 4 shows it as follows.

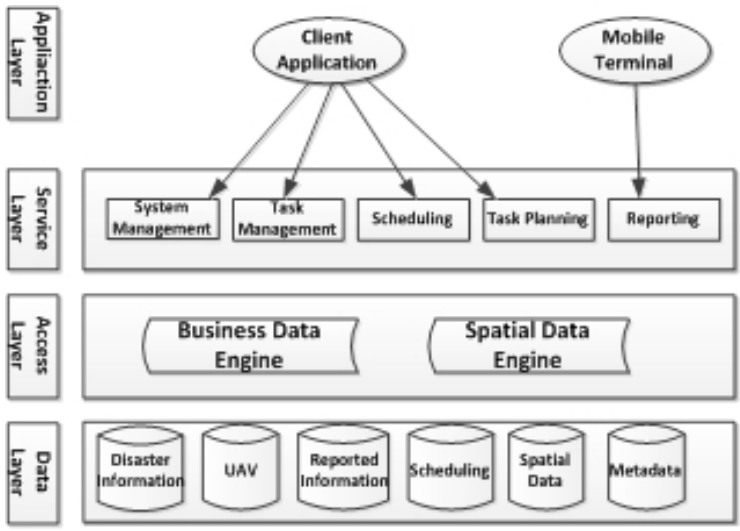

Figure 4 Software architecture

This software includes 4 tiers, namely data layer, access layer, service layer and application layer. The application layer is subdivided into a client application and mobile terminal APP. The client application is used to manage the information of UAVs and to schedule and plan the UAVs, and the mobile terminal APP is used by UAV manager to upload the status of their UAVs and their real-time position. The resident features, which stored as shape files, are employed to generate several key monitoring task regions via the hierarchical quadtree clustering method. The client application has been developed in C\# 2012 with ArcEngine 10.1. The figure 5 illustrates the client application.



Figure 5 Client Application 


\section{CONCLUSION}

In order to satisfy the time constrain of the UAV scheduling and planning for disaster relief or rescue, this paper developed a UAV tracking, scheduling and planning method. Towards the geological disaster, this method firstly collects the real-time positions of each UAV fleet via SMS and mobile APP. And, the network analysis module is used to estimate the arrival time for every UAV from their current site to the gathering site. Then, a few UAVs are scheduled to monitor all tasks, which are damaged and need to be monitored in the disaster area. In order to make the total finish time minimums, an optional task allocation scheme must be made. An approach to the UAV planning scheme is descripted in detail. So, the contribution of this paper includes: (1) designed a precise scheduling solution based on UAVs real-time location and status information, (2) designed a UAVs planning method based on the capability model, and minutely illustrate calculation steps.

However, there exist many problems to be improved in future work. (1) Only resident features are considered in outlining the task within a disaster area, the rivers, roads etc. should be taken into account. (2) The planning method only calculates the arrival time from their rally site to target task, the arrival time from their current site to rally site are ignored.

\section{ACKNOWLEDGEMENTS}

The work described in this article was supported by the National High Technology Research and Development Program of China (863 Program) under grant No. 2012AA121301, the National Natural Science Foundation of China under grant No. 41371366.

\section{REFERENCES}

O’ Rourke K., Bailey T., Hill R., et al, 2001. Dynamic Routing of Unmanned Aerial Vehicles Using Reactive Tabu Search. Military Operations Research Journal, 2001(6)pp. 5- 30.

Secrest, B., 2001. Traveling salesman problem for surveillance mission using particle swarm optimization. No. AFIT/GCE/ENG/01M-03). Air Force Inst of Tech WrightPatterson AFB OH School of Engineering and Management.

Alighanbari, M., 2004. Task assignment algorithms for teams of UAVs in dynamic environments. Doctoral dissertation, Massachusetts Institute of Technology.

Ye Y., 2005. Research on Mission Planning for Cooperative UCAVS. PHD, National University of Defense Technology.

Li X., Sun X., WANG D., et al, 2008. Dynamic UCAV mission assignment using genetic algorithm. Journal of System Simulation. 20(16)pp.4387-4389.

Zhang A., Shi Z., Liu H., et al, 2009. Cooperative task allocation for UCAV air-to-ground combat based on bayesian optimization algorithm. Electronics Optics \& C ontrol,16(1)pp.1-5.
Su, F., Yan, C., \& Lin-cheng, S., 2008. UAV cooperative multitask assignment based on ant colony algorithm. Acta Aeronautica et Astronautica Sinica, 29(S1)pp. S184-S191.

Tao, L, 2006. Research on distributed task allocation and coordination for multiple UCAVs cooperative mission control. Control Science and Engineering. vol. PHD ChangSha: National University of Defense Technology.

Tao L., Shen L., Zhu H., 2007. Distributed Tasks Allocation \& Coordination Technique of Multiple UCAVs for Cooperative Tasks. Acta Automatic Sinica, 3(7)pp.731-737

Yu Z., Chen Z., Zhou R., 2004. On dynamic resource scheduling based on genetic algorithm. Control and Decision, 19(11)pp. 1308-1311.

Stutzle, T., \& Hoos, H. 1997. MAX-MIN ant system and local search for the traveling salesman problem. In: IEEE Evolutionary Computation, pp. 309-314. 